\title{
Luminescence database - an update
}

\author{
N. C. Wilson ${ }^{1}$, C. M. MacRae ${ }^{1}$, A. Torpy ${ }^{1}$, M. Gaft ${ }^{2}$, J. Götze $^{3}$, C. Lenz ${ }^{4}$, L. Nasdala ${ }^{4}$, J. M. Hanchar ${ }^{5}$,
} and G. Barmarin ${ }^{6}$

${ }^{1}$ CSIRO Process Science and Engineering, Box 312, Clayton, VIC, 3169, Australia.

${ }^{2}$ Laser Distance Spectrometry Ltd., Petah Tikva, Israel

${ }^{3}$ Institut für Mineralogie, Technische Universität Bergakademie Freiberg, Germany

${ }^{4}$ Institut für Mineralogie und Kristallographie, Universität Wien, Austria

${ }^{5}$ Department of Earth Sciences, Memorial University of Newfoundland, St. John's, Canada

${ }^{6}$ www.fluomin.org/uk/accueil.php

Corresponding Author's e-mail address: nick.wilson@csiro.au

The luminescence database [1] was developed to provide a quick reference for identifying peaks in luminescence spectra, in similar fashion to KLM lines for x-rays or the RRUFF database for Raman lines. In the case of KLM lines, the characteristic x-ray energies are largely unaffected by bonding and so a table is relatively easy to compile. However, for luminescence the case is more complex, with the emission being sensitive to both material composition and structure of the host lattice. This complexity arises due to the luminescence signal being generated from a set of bonding influenced states such as conduction to valence band transitions, defect states and phonon modes [2]. Luminescence emission is generally grouped into intrinsic and extrinsic types, with intrinsic luminescence being native to the host material and extrinsic emission attributed to the presence of trace element impurities. These complications require the database to record not only the emitter of the line, but the host material in which it resides.

The initial database, generated from a literature survey of papers containing well characterized luminescence data, comprised over 70 minerals and a smaller number of synthetic materials, including semiconductors. With multiple entries for each structure, the database recorded over 1150 lines and bands, detailing the mineral name, associated chemical formula, charge on the ion or classification as intrinsic or extrinsic, and wavelength of energy of line observed. Wherever possible the sample temperature at which the emission line was recorded was included, as well as the technique used to excite and the instrument used to collect the luminescence. This was recorded as both temperature and polarisation effects can occur in a number of minerals/materials that lead to changes in relative peak intensity with wavelength. The publication reference for all entries was also noted so that further experimental and sample details can be examined. The database currently contains over 2700 lines.

The database was made public as a community resource available through the internet (http://www.csiro.au/luminescence/) and embedded within the freely available OpticalFit [3] software (Fig. 1). OpticalFit is a Windows-compatible software package for spectral fitting and peak deconvolution, and allows the database entries to be overlaid onto the spectra to aid in identification by choosing from a drop down list of structures, chemical formula and lines. In addition to recording line energies for particular minerals, the database now includes reference spectra generated using a number of techniques. This information greatly enhances the utility of the database, as it enables relative peak heights and efficiency of luminescence to be provided to the researcher.

Submissions to the database from the wider community have been welcomed, and since its inception, the database has grown considerably in size. M. Gaft, J. Götze, L. Nasdala, C. Lenz and J. M. Hanchar 
have most recently supplied collections of reference spectra and associated peak assignment collected across a wide range of minerals, both natural and synthetic, along with a set of related references detailing the samples and collection conditions. G. Barmarin, who has compiled a collection of luminescent minerals [4] and associated photoluminescence spectra, has contributed a large number of specimens from which we have collected cathodoluminescence (CL) reference spectra. Spectra from rutile and haematite, which are not normally thought of as CL active have also been included. We have been optimizing the collection of CL signals [5] from very weak emitters through hyper-spectral mapping and are growing the database to include reference spectra from such materials.

\section{References:}

[1] C.M. MacRae and N. C. Wilson, "Luminescence Database I - Minerals and Materials", Microscopy and Microanalysis, 14, 184-204, (2008)

[2] A. S. Marfunin, Spectroscopy, Luminescence and Radiation Centers in Minerals (Springer-Verlag, Berlin Heidelberg New York, ed. 1979).

[3] http://www.csiro.au/luminescence/opticalfit/index.html

[4] http://www.fluomin.org/uk/accueil.php

[5] N.C. Wilson, C.M. MacRae, and A. Torpy, Weak signal cathodoluminescence mapping, AMAS XII, Sydney 2013, p48-49

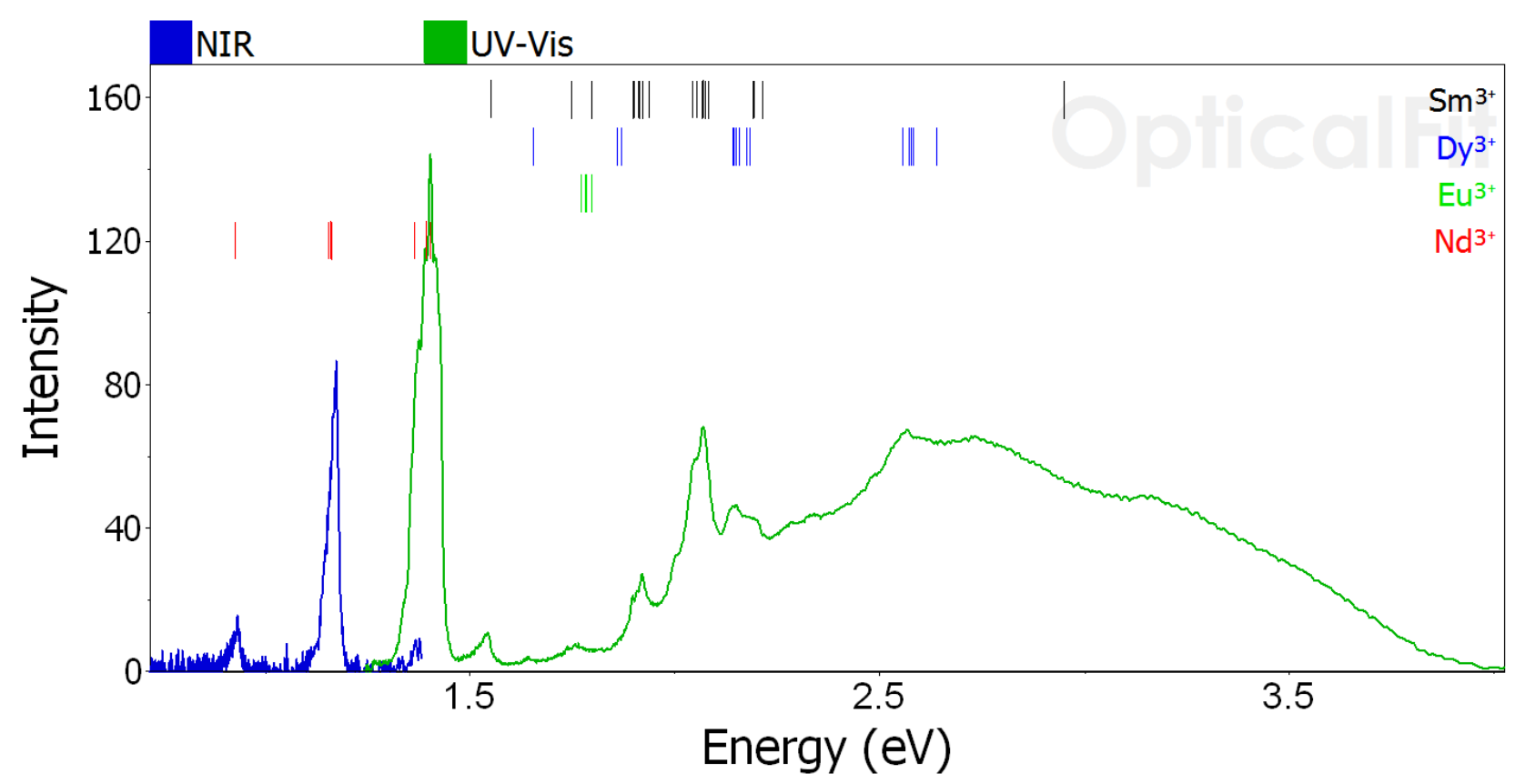

Figure 1 Optical fit showing a combined optical and near infra-red spectrum from an apatite sample. Colored vertical lines show database entries for Sm, Dy, Eu and Nd. 